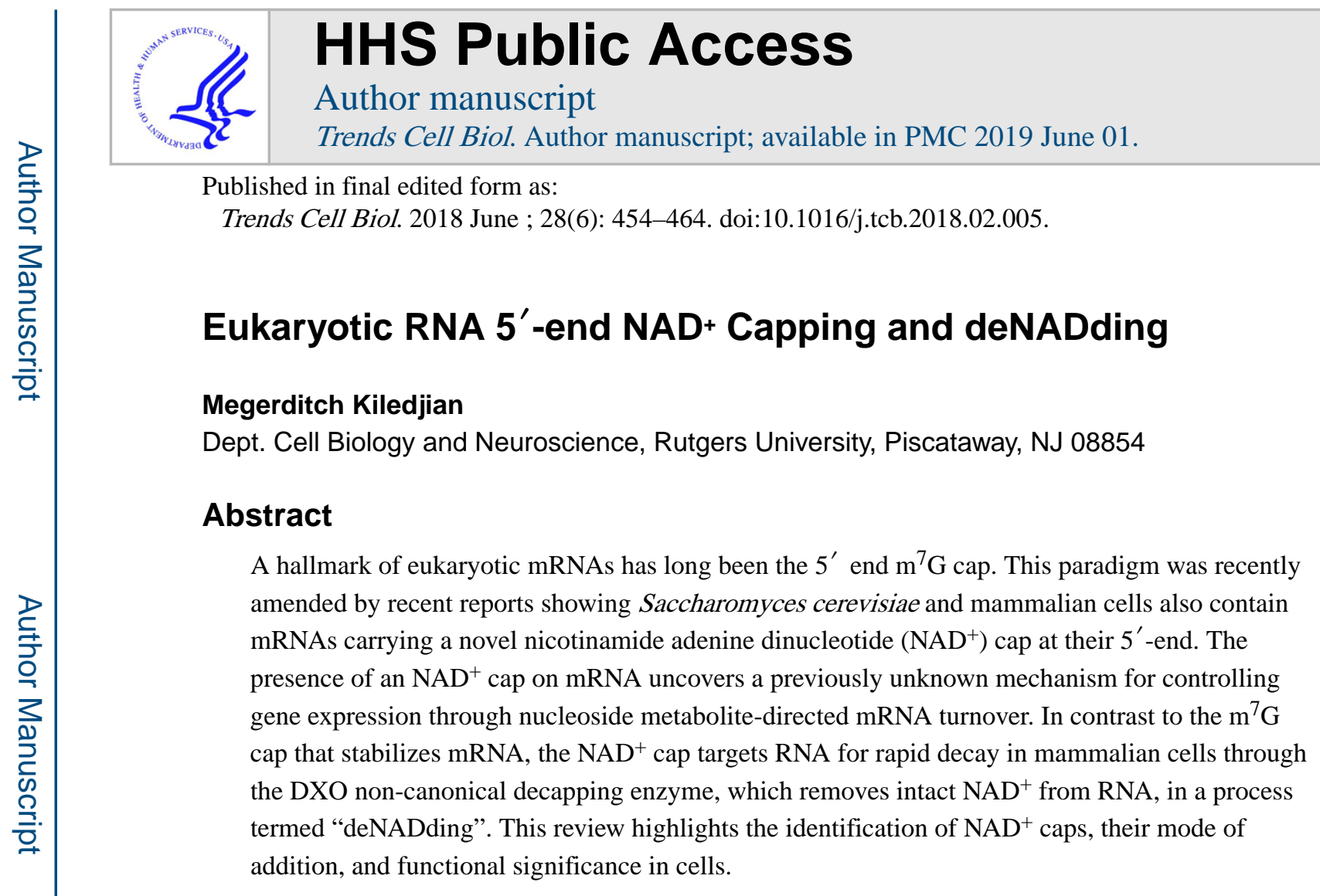

\title{
Overview
}

The triphosphorylated $5^{\prime}$ end of a nascent RNA polymerase II (RNAP II) transcript has long been known to undergo covalent modification to incorporate a $5^{\prime}-5^{\prime}$ linked $\mathrm{N}^{7}$-methyl guanosine $\left(\mathrm{m}^{7} \mathrm{G}\right)$ cap $[1,2]$. This modification imparts a layer of protection to the $5^{\prime}$ end of the mRNA from exonucleolytic attack $[3,4]$. The $\mathrm{m}^{7} \mathrm{G}$ cap can further be modified in some RNAs. A class of small nuclear RNAs can be hypermethylated in the cytoplasm on the $\mathrm{m}^{7} \mathrm{G}$ to generate the trimethylated $\mathrm{m}^{2,2,7} \mathrm{G}$ cap, which is essential for the reimport of these RNAs into the nucleus [5, 6]. Additional methylation can also occur on the $\mathrm{m}^{7} \mathrm{G}$ capped mRNA on the ribose $2^{\prime}$ hydroxyl position of the first and second transcribed nucleotides to generate the $2^{\prime}$ - $O$-methylated Cap1 and Cap2 mRNA forms, respectively [7, 8]. These modifications facilitate mRNA translation and contribute to distinguishing host from foreign viral mRNAs $[9,10]$. Moreover, a modification identified over forty years ago [8] consisting of methylations at the $N^{6}$ and $2^{\prime}-O$ positions $\left(\mathrm{m}^{6} \mathrm{~A}_{\mathrm{m}}\right)$ when the first nucleotide following the $\mathrm{m}^{7} \mathrm{G}$ cap is an adenosine, confers a key layer of epitranscriptomic regulatory information to the $5^{\prime}$ ends of $\mathrm{m}^{6} \mathrm{~A}_{\mathrm{m}}$ eukaryotic RNAs [11].

Recent findings in diverse organisms demonstrate epitranscriptomic regulation at the $5^{\prime}$-end cap extends beyond $\mathrm{m}^{6} \mathrm{~A}_{\mathrm{m}}$. A key breakthrough was the observation that bacterial RNA can carry the nucleoside-containing metabolite, nicotinamide adenine dinucleotide $\left(\mathrm{NAD}^{+}\right)$, on their $5^{\prime}$ end [12-14]. These studies established the foundation for recent reports

Publisher's Disclaimer: This is a PDF file of an unedited manuscript that has been accepted for publication. As a service to our customers we are providing this early version of the manuscript. The manuscript will undergo copyediting, typesetting, and review of the resulting proof before it is published in its final citable form. Please note that during the production process errors may be discovered which could affect the content, and all legal disclaimers that apply to the journal pertain. 
demonstrating $\mathrm{NAD}^{+}$can also decorate the $5^{\prime}$ end of eukaryotic RNAs in place of an $\mathrm{m}^{7} \mathrm{G}$ cap in Saccharomyces cerevisiae [15] and in human [16] cells. The NAD ${ }^{+}$cap can be introduced by RNA polymerase during transcription initiation with both bacterial RNAP and eukaryotic RNAP II in vitro [17-19], as well as in vivo in bacteria [17]. Thus $\mathrm{NAD}^{+}$cap addition (NADding) is likely to at least in part, occur with the use of $\mathrm{NAD}^{+}$as an initiation nucleotide in eukaryotic cells $[15,16]$. An important distinction between the canonical $\mathrm{m}^{7} \mathrm{G}$ cap and $\mathrm{NAD}^{+}$cap is apparent with mRNA stability and translation. Whereas the $\mathrm{m}^{7} \mathrm{G}$ cap promotes stability and translation, an $\mathrm{NAD}^{+}$cap in human cells targets the RNA for rapid decay and does not support translation. The decay is facilitated by the non-canonical decapping enzyme, DXO, which initiates degradation of the RNA by removing the NAD ${ }^{+}$ cap (deNADding) [16]. Recent advances in our understanding of NADding and deNADding are highlighted in this review with an emphasis on eukaryotic systems.

\section{Regulation of $5^{\prime}$ end cap in bacteria}

The initial demonstration that bacterial cells contain RNAs with a $5^{\prime}$-end cap was provided in 2009 [13, 14]. In particular, mass spectrometry of hydrolyzed Escherichia coli and Streptomyces venezuelae bacterial RNAs identified RNA species carrying $5^{\prime}$ end NAD $+[13]$ or $5^{\prime}$ end dephospho-coenzyme A (dpCoA) [14]. Nevertheless, since these $5^{\prime}$ end nucleoside metabolites were generated by hydrolyses of total RNA, the nature of the RNAs were not clear. The presence of NAD " "cap" at the $5^{\prime}$ end of bacterial RNA was further validated with the use of a chemo-enzymatic modification of a terminal nicotinamide to an alkyne moiety amendable to "click chemistry"-mediated biotinylation. Biotinylated RNAs are isolated by a streptavidin matrix and identified by high-throughput RNA sequencing in a process termed NAD-captureSeq [20] (Figure 1). NAD-captureSeq established the presence of $\mathrm{NAD}^{+}$caps at the $5^{\prime}$ end of bacterial RNAs and enabled identification of a subset of small regulatory RNAs that carry an $\mathrm{NAD}^{+}$-cap in Escherichia coli [12] (reviewed in [21]). Development of this approach proved an important tool to identify $\mathrm{NAD}^{+}$caps in other species.

\section{$\mathrm{NAD}^{+}$capping}

Two models can be envisioned for $\mathrm{NAD}^{+}$incorporation at the $5^{\prime}$ end of RNA, one being a transcriptional process and the second being a post-transcriptional process. Addition of the $\mathrm{NAD}^{+}$cap in bacteria proceeds through the former mechanism where $\mathrm{NAD}^{+}$serves as a noncanonical initiating nucleotide (NCIN) in place of ATP as the first nucleotide [17]. The NCIN model for $\mathrm{NAD}^{+}$capping is supported by evidence that bacterial RNAP can use NAD ${ }^{+}$as an initiating nucleotide in place of ATP in vitro [17-19]. Furthermore, promoter sequence determinants upstream of the transcription start site can influence the efficiency by which $\mathrm{NAD}^{+}$can serve as an NCIN in vitro [17]. A direct correlation is observed between the promoter sequence determinants for use of $\mathrm{NAD}^{+}$as an $\mathrm{NCIN}$ in vitro and the amount of $\mathrm{NAD}^{+}$-capped RNA detected in E. coli [17]. Correlations between the effects of promoter sequence on the efficiency of use of $\mathrm{NAD}^{+}$as an NCIN in vitro and the amounts of $\mathrm{NAD}^{+}-$ capped RNA generated in vivo provide strong support for the proposal that most (if not all) $\mathrm{NAD}^{+}$-capped RNA in bacterial cells is generated by use of NAD ${ }^{+}$as an NCIN [17]. 
Interestingly, the capacity of bacterial RNAP to incorporate NCINs is not restricted to NAD

${ }^{+}$. Transcription initiation through the adenosine residue of nucleoside metabolites (FIGURE

2 ) is also observed in vitro including the reduced form of $\mathrm{NAD}^{+}(\mathrm{NADH})$, flavin adenine diphosphate (FAD) and desphospho coenzyme $\mathrm{A}(\mathrm{dpCoA})$, as well as uridine containing metabolites including uridine diphosphate glucose (UDP-glucose), and uridine diphosphate $\mathrm{N}$-acetylglucosamine (UDP-GlcNAc) [17-19]. To date, dpCoA is the only nucleoside metabolite other than $\mathrm{NAD}^{+}$, detected at the $5^{\prime}$ end of bacterial RNA [14].

\section{NudC and deNADding of bacterial RNA}

In mammals, the protective $5^{\prime}$ end $\mathrm{m}^{7} \mathrm{G}$ cap is removed by a subclass of nucleoside diphosphate linked to another moiety $\mathrm{X}$ (Nudix) hydrolase family of proteins including Dcp2, Nudt3 and Nudt16 known to function as decapping enzymes in cells, and Nudt2, Nudt12, Nudt15, Nudt17 and Nudt19 with in vitro decapping activity [22]. These decapping enzymes can remove the cap structure to generate a $5^{\prime}$ end monophosphorylated RNA substrate (Figure 3A) that is subjected to subsequent exonucleolytic decay. Similarly, in bacteria, the RppH Nudix protein can remove the first two phosphates from the $5^{\prime}$ end of triphosphorylated RNA to generate a monophosphorylated 5' end RNA [23] that can serve as a substrate for RNaseE-directed decay [24]. Another bacterial Nudix protein, NudC, previously shown to hydrolase free $\mathrm{NAD}(\mathrm{H})$ into nicotinamide mononucleotide $(\mathrm{NMN}(\mathrm{H}))$ plus AMP [25] possesses deNADding activity that hydrolyzes NAD ${ }^{+}$-capped RNA to NMN plus $5^{\prime}$ monophosphate RNA [12] (Figure 3B). The significance of NudC deNADding activity was demonstrated in $E$. coli harboring a deleted $N u d C$ gene, where the steady state accumulation of $\mathrm{NAD}^{+}$-capped small RNAs increased [12] as did their stability [17]. These findings indicate the $\mathrm{NAD}^{+}$cap functions in a role analogous to the eukaryotic $\mathrm{m}^{7} \mathrm{G}$ cap to stabilize RNA in bacteria and NudC is a deNADding enzyme that removes the protective $\mathrm{NAD}^{+}$cap to promote decay. Furthermore, in vitro data shows that NudC can remove dpCoA from the $5^{\prime}$ end of RNA [17], suggesting a potential role for NudC in the removal of dpCoA caps in vivo. It is likely now a question of when, rather than if, additional nucleoside metabolite $5^{\prime}$ end caps are identified, which in turn will raise new questions of whether the new caps are also removed by NudC or removed by other as yet uncharacterized enzymes.

\section{Regulation of non-canonical $5^{\prime}$ end cap in mammalian cells}

Addition and removal of the $\mathrm{m}^{7} \mathrm{G}$ cap has been extensively studied in the last four decades with little emphasis on alternative caps. In eukaryotes, the first $5^{\prime}$ mRNA cap to be identified, the $\mathrm{m}^{7} \mathrm{G}$ cap [1, 2], functions to stabilize RNA and facilitate mRNA translation as well as contribute to RNA splicing and RNA transport [26]. Additional caps have subsequently been described including the $N^{6}$ - and $2^{\prime}$ - $O$-methylated $\mathrm{m}^{6} \mathrm{~A}_{\mathrm{m}}$. The $\mathrm{m}^{6} \mathrm{~A}_{\mathrm{m}}$ cap confers a key layer of regulatory information to $5^{\prime}$ ends of mRNA because this cap is less susceptible to decapping by the Dcp2 decapping enzyme [11]. Furthermore, capping does not always proceed to completion in eukaryotic cells. Incomplete caps lacking the $\mathrm{N}^{7}$ methyl moiety or lacking a cap altogether, can also be generated. These incomplete caps are detected and cleared by a $5^{\prime}$-end quality control $\left(5^{\prime} \mathrm{QC}\right)$ mechanism employing the DXO family of proteins [27-29] (Figure 3A). Existence of diverse mRNA caps underscores the 
significance of regulatory mechanisms that incorporate or remove the $5^{\prime}$-end cap and their influence on controlling gene expression.

\section{$\mathrm{NAD}^{+}$capping}

Recently, the NAD-captureSeq approach was used to demonstrate $\mathrm{NAD}^{+}$caps extend beyond bacterial RNA and are also present in human cells [16]. The majority of NAD ${ }^{+}$ capped mRNAs overlap with canonical $\mathrm{m}^{7} \mathrm{G}$-capped mRNAs indicating mRNAs can be present as two distinct populations in cells that differ in the state of their $5^{\prime}$-end cap. One population carries the canonical $\mathrm{m}^{7} \mathrm{G}$-cap, while the other, estimated to comprise $\sim 1-6 \%$ of a respective mRNA [16], harbors an $\mathrm{NAD}^{+}$cap. Interestingly, distinctions between the reads obtained from NAD-captureSeq and standard RNA-seq are also evident. For example, while the proportion of small nuclear RNAs (snRNAs) are higher in NAD ${ }^{+}$-capped RNA populations, the proportion of another class of $\mathrm{NAD}^{+}$-capped RNAs consisting of antisense transcripts, are significantly lower than that in $\mathrm{m}^{7} \mathrm{G}$-capped RNA [16] suggestive of transcript preference in $\mathrm{NAD}^{+}$capping (see below).

\section{Post-transcriptional NAD ${ }^{+}$capping in mammalian cells}

The demonstration that eukaryotic RNAP II can use $\mathrm{NAD}^{+}$as an initiating nucleotide in vitro [17] suggests that $\mathrm{NAD}^{+}$-capped mRNAs detected in yeast [15] and human cells [16] are also generated by use of $\mathrm{NAD}^{+}$as an NCIN by RNAP II. However, a similar transcriptional NCIN model cannot account for all mammalian $\mathrm{NAD}^{+}$-capped RNAs. In particular, the existence of an $\mathrm{NAD}^{+}$cap on mammalian snoRNAs and the related small Cajal body RNAs (scaRNAs) indicates a post-transcriptional mechanism is also involved [16]. SnoRNAs and scaRNAs function as guide RNAs in the pseudouridylation and methylation of ribosomal RNAs and U-rich small nuclear RNAs, respectively [30, 31]. They are predominantly encoded within host gene introns and generated as exonucleolyticresistant ribonucleoprotein complexes lacking a modification at their $5^{\prime}$ end [30-32]. The presence of $\mathrm{NAD}^{+}$caps on intronic RNAs strongly suggest $\mathrm{NAD}^{+}$caps can be added to the $5^{\prime}$ end of RNAs post-transcriptionally and supports the existence of an $\mathrm{NAD}^{+}$capping mechanism in addition to the NCIN model in mammalian cells.

Addition of the canonical $\mathrm{m}^{7} \mathrm{G}$ cap to the $5^{\prime}$ end of pre-mRNAs occurs cotranscriptionally by the combination of three enzymatic activities: a triphosphatase activity, a guanylyltransferase activity, and a methyltransferase activity [33, 34]. Three different proteins carry out the distinct activities in yeast, while the triphosphatase and guanylyltransferase activities are carried out by a single bifunctional capping enzyme in mammals [35]. A multistep cytoplasmic recapping of $5^{\prime}$ end monophosphorylated RNA has also been reported [36]. Although the mechanism for $\mathrm{NAD}^{+}$cap addition is unknown, at least two distinct modes of NADding can be envisioned. First, nicotinamide or its derivative, could be linked onto an RNA containing a 5' adenosine. Second, addition of an intact NAD ${ }^{+}$molecule onto the $5^{\prime}$ end of an RNA regardless of the first nucleotide on the RNA. Based on current identified RNA ligases [37] a simple ligation of $\mathrm{NAD}^{+}$onto sno/scaRNA appears unlikely as the mechanism for NADding. Whether NADding occurs through a currently unknown RNA ligase or through a multiprotein sequential process is unknown. Identification of the NADding mechanism and the enzyme(s) involved will be an important 
step forward in our understanding of $\mathrm{NAD}^{+}$-capping and provide insight into potential modes of NADding regulation.

\section{DXO and deNADding of mammalian RNA}

The DXO family of proteins are non-canonical decapping enzymes, that remove the entire cap structure of capped, or incompletely capped, RNA [28] (Figure 3A). This activity is distinct from that of the "traditional" decapping enzymes, Dcp2 [38-40], Nudt3 [41] and Nudt16 [42] that cleave the cap within the triphosphate linkage (Figure 3A). The ability of DXO to function on incomplete cap structures suggested a potential role for this enzyme in the removal of $\mathrm{NAD}^{+}$-caps. Consistent with this notion, DXO, but not Dcp2, exhibits deNADding activity on RNA transcripts in vitro [16]. The deNADding activity of DXO results in removal of the entire $\mathrm{NAD}^{+}$moiety leaving a $5^{\prime}$ end monophosphate RNA which is mechanistically distinct from that of NudC-mediated deNADding, which results in the conversion of $\mathrm{NAD}^{+}$-capped RNA to NMN plus $5^{\prime}$ monophosphate RNA (Figure 3B). Notably, the activity of DXO on NAD ${ }^{+}$-capped RNA is $\sim 6$ fold more efficient than the activity on $\mathrm{m}^{7} \mathrm{G}$-capped RNA, suggesting deNADding is likely a prominent activity for DXO in cells.

Two lines of evidence support a role for DXO in modulating levels of $\mathrm{NAD}^{+}$-capped RNAs in cells. The first is from transfected generic RNAs. NAD ${ }^{+}$-capped RNAs transfected into HEK293T cells lacking DXO are more stable than NAD ${ }^{+}$-capped RNAs transfected into cells lacking the Dcp2 decapping enzyme, which does not cleave $\mathrm{NAD}^{+}$capped RNA [16]. Second, $\mathrm{NAD}^{+}$-capped RNA levels are elevated in cells lacking DXO, with the most prominent being the sno/scaRNAs, which can increase up to nine fold. Together, these findings indicate DXO is a deNADding enzyme in cells that specifically targets a subset of $\mathrm{NAD}^{+}$-capped RNAs.

The established role for the DXO family of proteins in $5^{\prime} \mathrm{QC}$ [43] raises an interesting question. Is $\mathrm{NAD}^{+}$capping a result of random aberrant incorportation of $\mathrm{NAD}^{+}$as an initiating nucleotide that is subjected to the DXO $5^{\prime} \mathrm{QC}$ mechanism? Although this remains a formal posibility, several observations argue against nonspecific $\mathrm{NAD}^{+}$-capping as the promonent pathway. First, random incorporation of $\mathrm{NAD}^{+}$caps would be expected at all adenosine transcription initiation sites, resulting in proportional random distribution of NAD ${ }^{+}$caps at the $5^{\prime}$ end of RNAs. However, this is not the case as subsets of RNA classes contain either higher or lower proportions of $\mathrm{NAD}^{+}$caps relative to the same class of RNAs containing canonical caps [16]. The nonuniform distribution of $\mathrm{NAD}^{+}$-capped RNAs supports the premise that $\mathrm{NAD}^{+}$capping is not a stochastic event uniformly $\mathrm{NAD}^{+}$capping all RNAs. A comparison of cellular $\mathrm{NAD}^{+}$levels under parameters that increase or decrease $\mathrm{NAD}^{+}$levels, to that of potential corresponding changes in $\mathrm{NAD}^{+}$-capped RNA levels in cells can begin to address this point. An outcome of 1:1 uniformity between cellular NAD ${ }^{+}$ and $\mathrm{NAD}^{+}$-caps would be predicted from random incorporation, while variances between the two would be observed with specific $\mathrm{NAD}^{+}$-capping. Second, and perhaps most significantly, identification of $\mathrm{NAD}^{+}$-caps on a subset of sno/scaRNAs demonstrates $\mathrm{NAD}^{+}$capping is an orchestrated and not a random process, at least for this subset of RNAs. 


\section{NAD+ cap promotes RNA decay in human cells}

In contrast to the $\mathrm{m}^{7} \mathrm{G}$ cap, which stabilizes the $5^{\prime}$ end of mRNAs, the $5^{\prime} \mathrm{NAD}^{+}$-cap promotes decay of RNAs it is attached to. $\mathrm{NAD}^{+}$-capped and polyadenylated luciferase mRNAs transfected into HEK293T cells are less stable than the same mRNAs lacking a $5^{\prime}$ end cap [16]. Therefore, the $\mathrm{NAD}^{+}$-cap does not serve as a simple passive marker on the $5^{\prime}$ end of RNAs, but rather functions to actively recruit and confer deNADding and decay. Moreover, the decay of an $\mathrm{NAD}^{+}$-capped mRNA is mediated through DXO as indicated by the equivalent stability of an $\mathrm{NAD}^{+}$-capped luciferase $\mathrm{mRNA}$ to that of an $\mathrm{m}^{7} \mathrm{G}$-capped luciferase mRNA in DXO-knockout cells. It appears the $\mathrm{NAD}^{+}$cap fulfills a function similar to mRNA $3^{\prime}$ terminal uridylation. The uridine tract on the $3^{\prime}$ end of an mRNA promotes its decay [44] by is susceptibility to the Dis 3 L $23^{\prime}$ exonuclease $[45,46]$ as well as by recruiting the mRNA decapping complex $[47,48]$. By analogy, the NAD ${ }^{+}$-cap serves as a $5^{\prime}$-end tag to recruit DXO and facilitate the demise of the mRNA (Figure 4). The deNADding activity of DXO hydrolyzes and releases $\mathrm{NAD}^{+}$, leaving the $5^{\prime}$ monophosphorylated mRNA molecule which can further be degraded by the intrinsic $5^{\prime}-3^{\prime}$ exonucleolytic activity of DXO [28].

An important question remains regarding the physiological function of the $\mathrm{NAD}^{+}$cap, to which the $\mathrm{NAD}^{+}$capped sno/scaRNAs may provide a clue. Intronic sno/scaRNAs are generated by their exonucleolytic resistant property following release of the spliced intron [49] and are therefore expected to be refractory to exonucleolytic decay as exemplified by snoRNA associated long noncoding RNAs (sno-lncRNAs) which consist of stable intronic RNAs flanked on both termini by snoRNAs [50]. Addition of an $\mathrm{NAD}^{+}$cap could provide a mechanism to recruit the DXO exonuclease to promote their decay. It is also plausible that $5^{\prime}$ end stable decay intermediates of mRNAs that originally initiated as a $\mathrm{m}^{7} \mathrm{G}$ capped mRNA, could potentially be $\mathrm{NAD}^{+}$capped to further promote their decay (Figure 4). However, it should be noted that this latter scenario is a model that still requires experimental verification.

\section{$\mathrm{NAD}^{+}$cap does not support translation in human cells}

A prominent feature of the $\mathrm{m}^{7} \mathrm{G}$ cap is its role in translation initiation by recruitment of the initiation complex onto the mRNA [51]. Translation initiation complex assembly onto an mRNA can also occur by $\mathrm{m}^{7} \mathrm{G}$ cap-independent mechanisms [52]. The capacity of the NAD ${ }^{+}$cap in translation was assessed by transfection of $\mathrm{NAD}^{+}$-capped and polyadenylated luciferase mRNA into HEK293T cells where the level of translation from this mRNA was no more than the background level of translation from an identical uncapped RNA [16]. These findings show that the $\mathrm{NAD}^{+}$cap does not support translation of the exogenous mRNA used. However, these studies cannot rule out the possibility that the $\mathrm{NAD}^{+}$cap may promote alternative cap independent mechanisms within the proper endogenous mRNA context. NAD-captureSeq of polysomal mRNA fractions will be necessary to begin addressing cap-alternative modes of potential $\mathrm{NAD}^{+}$-capped mRNA translation. 


\section{Regulation of $5^{\prime}$ end non-canonical cap in yeast}

$\mathrm{NAD}^{+}$-capped mRNAs were also recently identified in the yeast Saccharomyces cerevisiae using the NAD-captureSeq approach [15] reinforcing the widespread utilization of NAD ${ }^{+}$ caps in diverse organisms.

\section{NAD+ capping}

Addition of the $\mathrm{NAD}^{+}$cap in yeast appears to predominantly occur through the NCIN model of NADding [15], indicating transcriptional incorporation of an $\mathrm{NAD}^{+}$cap is a prevalent theme across multiple kingdoms. Interestingly, the extent of $\mathrm{NAD}^{+}$capped mRNA is comparable between yeast and human cells. Estimates of $\mathrm{NAD}^{+}$-capped mRNA in yeast range from $1-5 \%$ of a given mRNA population [15] which is similar to mammalian cells under normal growth conditions [16]. However, $\mathrm{NAD}^{+}$-capped mRNAs increase in yeast cells grown in synthetic media [15] an indication that the levels of $\mathrm{NAD}^{+}$-capped mRNA detected under optimal growth conditions may be an underestimation. The results also imply cellular stress may contribute to the extent of $\mathrm{NAD}^{+}$capped mRNA. Whether additional cellular or environmental conditions alter levels of $\mathrm{NAD}^{+}$capped mRNA in yeast remains unknown. Beyond nuclear encoded mRNAs, gene ontology analysis of the $\mathrm{NAD}^{+}$-capped transcripts in yeast uncovered an enrichment of mitochondrial mRNAs [15]. Mitochondrial transcription begins with an adenosine as the first nucleotide [53], suggesting $\mathrm{NAD}^{+}$capping of mitochondrial mRNA through the NCIN mechanism may provide an avenue of coordinating the fate of RNA with cellular metabolism.

\section{Removal of the $\mathrm{NAD}^{+}$cap}

At present the functional role of the $\mathrm{NAD}^{+}$cap in yeast has not been determined. Whether $\mathrm{NAD}^{+}$caps stabilize mRNAs as observed in bacteria [17], destabilize RNA as observed in mammals [16], and/or impact translation, is unknown. Nevertheless, insight into potential deNADding enzymes exist. Yeast contain two members of the DXO family of proteins, Rai1p [54] and Dxo1p [27]. Rai1p forms a heterodimer with the nuclear $5^{\prime}-3^{\prime}$ exoribonuclease, Rat1p [54, 55], which degrades RNAs carrying a 5' monophosphate. Railp can generate $5^{\prime}$ monophosphorylated RNA substrates for Rat $1 \mathrm{p}$ by catalyzing the removal of an incomplete cap lacking the $\mathrm{N}^{7}$ methyl moiety [29]. Dxo1p can remove an incomplete cap lacking the $\mathrm{N}^{7}$ methyl moiety and similar to DXO, contains intrinsic $5^{\prime}-3^{\prime}$ exoribonuclease activity to degrade the decapped mRNA [27]. Similar to mammalian DXO, the yeast Schizosaccharomyces pombe Railp and Kluyveromyces lactis Dxo1p exhibit deNADding activity in vitro [16]. Furthermore, the deNADding activity of both proteins is more robust than their respective incomplete cap decapping activities. Whether one or both proteins function as deNADding enzymes in yeast cells, and whether they target specific subclasses of $\mathrm{NAD}^{+}$-capped RNAs will be important areas to address.

The yeast enhancer of decapping protein, $\mathrm{Edc} 3$, is an $\mathrm{NAD}(\mathrm{H})$ binding protein and reported to cleave free $\mathrm{NAD}^{+}$in vitro at excess protein concentrations [56]. These observations suggest Edc3 may function as a deNADding enzyme. However, $\mathrm{NAD}(\mathrm{H})$ binding influences the cellular localization of Edc3 in yeast cells [56] implying that similar to the sirtuin family of DNA deacetylases that require $\mathrm{NAD}^{+}$binding for their function [57, 58], $\mathrm{NAD}(\mathrm{H})$ may be 
an essential Edc3 cofactor rather than a substrate on the $5^{\prime}$ of an mRNA. Whether Edc3 utilizes $\mathrm{NAD}(\mathrm{H})$ as a cofactor or functions as a deNADding enzyme remains to be determined.

\section{Potential link between NAD+ capping and cellular metabolism}

RNA-binding proteins have been known to bridge RNA metabolism and cellular metabolism since the 1990's with the tricarboxylic acid (TCA) cycle enzyme, aconitase, being one of the best studied examples [59]. In the absence of iron, the aconitase enzyme is endowed with RNA-binding properties to bind a stem loop structure and influence mRNA stability and translation $[60,61]$. The glyceraldehyde-3-phosphate dehydrogenase (GAPDH) glycolytic enzyme is also an RNA-binding protein and its binding activity is modulated by the presence of NAD ${ }^{+}$. NAD ${ }^{+}$inhibits the RNA binding property of GAPDH, but is necessary for GAPDH enzymatic activity [62]. The identification of $\mathrm{NAD}^{+}$capped RNAs indicates the connection between RNA fate and cellular metabolism may extend beyond just RNAbinding proteins and include the RNA itself through its $5^{\prime}$ end cap. The fact that DXO regenerates an intact $\mathrm{NAD}^{+}$from an mRNA is suggestive of an intimate link between these processes. Cellular $\mathrm{NAD}^{+}$levels are altered in response to energy stresses, such as glucose deprivation [63], fasting [64, 65] and caloric restriction [66] indicating the nutrient state of a cell influences $\mathrm{NAD}^{+}$concentrations. It is highly probable that such changes would impact $\mathrm{NAD}^{+}$capping. Since nutrient deprivation can manifest altered mRNA stability [67-69] and $\mathrm{NAD}^{+}$caps promote mRNA decay [16], a tantalizing possibility exists that the energetic state of a cell can impact $\mathrm{NAD}^{+}$-capping and in turn mRNA turnover. Such a connection could position the $\mathrm{NAD}^{+}$cap as an important rheostat influencing the stability of transcripts involved in cellular energetics. The relative higher level of $\mathrm{NAD}^{+}$-capped RNA observed in stationary phase bacteria [17] and yeast grown in synthetic media [15] support such a possibility.

\section{Concluding Remarks}

The discovery of the $\mathrm{m}^{7} \mathrm{G}$ cap on mRNAs over four decades ago opened a new and exciting area of research for RNA biology and the control of mRNA fate through its addition and removal, that still continues to date. Discovery of a new RNA cap, the NAD ${ }^{+}$cap, provides yet another layer of complexity and excitement to the field. We have only begun delineating the role of the $\mathrm{NAD}^{+}$cap in mRNA turnover with much of the molecular details for the function and outcome of the $\mathrm{NAD}^{+}$cap unknown. Other than transcriptional initiation, how is the $\mathrm{NAD}^{+}$cap added and what are the enzyme(s) involved? Does the function of an $\mathrm{NAD}^{+}$ cap extend beyond RNA turnover? Although an $\mathrm{NAD}^{+}$-capped exogenous luciferase mRNA was not translated when transfected into cells [16], could endogenous NAD ${ }^{+}$-capped mRNA be translated by cap-independent mechanisms [70], bypassing the need for a $\mathrm{m}^{7} \mathrm{G}$ cap? Is there an equivalent to the eIF4E $\mathrm{m}^{7} \mathrm{G}$ cap binding protein for the $\mathrm{NAD}^{+}$cap? If so, would it modulate mRNA turnover, translation or localization?

Identification of the DXO family of proteins as deNADding enzymes is a major step towards understand the physiological regulation of $\mathrm{NAD}^{+}$capping. However, based on the precedent in mammalian cells where there are multiple $\mathrm{m}^{7} \mathrm{G}$ decapping enzymes [22], additional 
deNADding enzymes with selective specificities are expected. Moreover, all the studies thus far have focused on $\mathrm{NAD}^{+}$capping. RNAP can also incorporate the reduced form of $\mathrm{NAD}^{+}$, NADH as the first nucleotide [17]. Are RNAs capped with NADH and considering the contrasting energetic states of $\mathrm{NAD}^{+}$and $\mathrm{NADH}$, do RNAs with these modifications have distinct functions? Beyond the $\mathrm{NAD}^{+}$cap, a pressing question is whether additional nucleoside metabolites are used as a $5^{\prime}$ end cap. Polymerases can incorporate NCIN's in vitro [71] and E. coli and $S$. venezuelae RNAs can contain dpCoA at their $5^{\prime}$ end [14]. It is highly likely that the $\mathrm{NAD}^{+}$cap is only the beginning of a new revolution in $5^{\prime}$ end capping (See Outstanding Questions). We are at an analogous position with nucleoside metabolite capping of mRNA as we were in the 1970's with the discovery of the $\mathrm{m}^{7} \mathrm{G}$ cap. The road ahead will undoubtedly be just as exciting and full of surprising new discoveries to be uncovered.

\section{Outstanding Questions}

What determines the proportion of a given mRNAs capacity to be capped with an $\mathrm{NAD}^{+}$, rather than $\mathrm{m}^{7} \mathrm{G}$ cap and does the percentage of $\mathrm{NAD}^{+}$cap on any given mRNA population change with intracellular or extracellular stimuli?

Capping of intronic RNAs must proceed through a post-transcriptionally NAD ${ }^{+}$ capping mechanism. What are the enzymes and the pathway involved?

Do cells possess an $\mathrm{NAD}^{+}$cap-binding protein (NCBP) and is the state of the $\mathrm{NAD}^{+}$cap modulated by such a protein?

Do eukaryotic cells also contain additional $5^{\prime}$-end nucleoside metabolite caps other than $\mathrm{NAD}^{+}$and do they provide a conduit between RNA metabolism and cellular energetics?

\section{Acknowledgments}

Insightful suggestions and critical reading of the manuscript by Drs. Xinfu Jiao and Bryce Nickels are greatly appreciated. This work was supported by NIH grant GM067005.

\section{References}

1. Furuichi Y, et al. Reovirus messenger RNA contains a methylated, blocked $5^{\prime}$-terminal structure: m-7G(5')ppp(5')G-MpCp. Proc Natl Acad Sci U S A. 1975; 72(1):362-6. [PubMed: 1054511]

2. Wei CM, et al. Methylated nucleotides block $5^{\prime}$ terminus of HeLa cell messenger RNA. Cell. 1975; 4(4):379-86. [PubMed: 164293]

3. Furuichi Y, et al. 5'-Terminal structure and mRNA stability. Nature. 1977; 266(5599):235-9. [PubMed: 557727]

4. Wang Z, Kiledjian M. Functional Link between the Mammalian Exosome and mRNA Decapping. Cell. 2001; 107(6):751-762. [PubMed: 11747811]

5. Hamm J, Mattaj IW. Monomethylated cap structures facilitate RNA export from the nucleus. Cell. 1990; 63(1):109-18. [PubMed: 2208274]

6. Lamond AL. The trimethyl-guanosine cap is a nuclear targeting signal for snRNPs. Trends Biochem Sci. 1990; 15(12):451-2. [PubMed: 2150241]

7. Adams JM, Cory S. Modified nucleosides and bizarre $5^{\prime}$-termini in mouse myeloma mRNA. Nature. 1975; 255(5503):28-33. [PubMed: 1128665] 
8. Wei C, et al. N6, $\mathrm{O}^{\prime}{ }^{\prime}$-dimethyladenosine a novel methylated ribonucleoside next to the $5^{\prime}$ terminal of animal cell and virus mRNAs. Nature. 1975; 257(5523):251-3. [PubMed: 1161029]

9. Daffis $\mathrm{S}$, et al. $2^{\prime}$-O methylation of the viral mRNA cap evades host restriction by IFIT family members. Nature. 2010; 468(7322):452-6. [PubMed: 21085181]

10. Devarkar SC, et al. Structural basis for $\mathrm{m} 7 \mathrm{G}$ recognition and $2^{\prime}$-O-methyl discrimination in capped RNAs by the innate immune receptor RIG-I. Proc Natl Acad Sci U S A. 2016; 113(3):596-601. [PubMed: 26733676]

11. Mauer J, et al. Reversible methylation of m6Am in the $5^{\prime}$ cap controls mRNA stability. Nature. 2017; 541(7637):371-375. [PubMed: 28002401]

12. Cahova $\mathrm{H}$, et al. NAD captureSeq indicates NAD as a bacterial cap for a subset of regulatory RNAs. Nature. 2015; 519(7543):374-7. [PubMed: 25533955]

13. Chen YG, et al. LC/MS analysis of cellular RNA reveals NAD-linked RNA. Nat Chem Biol. 2009; 5(12):879-81. [PubMed: 19820715]

14. Kowtoniuk WE, et al. A chemical screen for biological small molecule-RNA conjugates reveals CoA-linked RNA. Proc Natl Acad Sci U S A. 2009; 106(19):7768-73. [PubMed: 19416889]

15. Walters RW, et al. Identification of NAD+ capped mRNAs in Saccharomyces cerevisiae. Proc Natl Acad Sci U S A. 2017; 114(3):480-485. [PubMed: 28031484]

16. Jiao X, et al. $5^{\prime}$ End Nicotinamide Adenine Dinucleotide Cap in Human Cells Promotes RNA Decay through DXO-Mediated deNADding. Cell. 2017; 168(6):1015-1027. e10. [PubMed: 28283058]

17. Bird JG, et al. The mechanism of RNA $5^{\prime}$ capping with NAD+, NADH and desphospho-CoA. Nature. 2016; 535(7612):444-7. [PubMed: 27383794]

18. Julius C, Yuzenkova Y. Bacterial RNA polymerase caps RNA with various cofactors and cell wall precursors. Nucleic Acids Res. 2017; 45(14):8282-8290. [PubMed: 28531287]

19. Malygin AG, Shemyakin MF. Adenosine, NAD and FAD can initiate template-dependent RNA synthesis catalyzed by Escherichia coli RNA polymerase. FEBS Lett. 1979; 102(1):51-4. [PubMed: 222618]

20. Winz ML, et al. Capture and sequencing of NAD-capped RNA sequences with NAD captureSeq. Nat Protoc. 2017; 12(1):122-149. [PubMed: 27977022]

21. Jaschke A, et al. Cap-like structures in bacterial RNA and epitranscriptomic modification. Curr Opin Microbiol. 2016; 30:44-9. [PubMed: 26779928]

22. Grudzien-Nogalska, E., Kiledjian, M. Wiley Interdiscip Rev RNA. 2016. New Insights into Decapping Enzymes and Selective mRNA Decay.

23. Deana A, et al. The bacterial enzyme RppH triggers messenger RNA degradation by $5^{\prime}$ pyrophosphate removal. Nature. 2008; 451(7176):355-8. [PubMed: 18202662]

24. Bouvier M, Carpousis AJ. A tale of two mRNA degradation pathways mediated by RNase E. Mol Microbiol. 2011; 82(6):1305-10. [PubMed: 22074454]

25. Frick DN, Bessman MJ. Cloning, purification, and properties of a novel NADH pyrophosphatase. Evidence for a nucleotide pyrophosphatase catalytic domain in MutT-like enzymes. J Biol Chem. 1995; 270(4):1529-34. [PubMed: 7829480]

26. Ramanathan A, et al. mRNA capping: biological functions and applications. Nucleic Acids Res. 2016; 44(16):7511-26. [PubMed: 27317694]

27. Chang JH, et al. Dxo1 is a new type of eukaryotic enzyme with both decapping and $5^{\prime}-3^{\prime}$ exoribonuclease activity. Nat Struct Mol Biol. 2012; 19(10):1011-7. [PubMed: 22961381]

28. Jiao X, et al. A mammalian pre-mRNA $5^{\prime}$ end capping quality control mechanism and an unexpected link of capping to pre-mRNA processing. Mol Cell. 2013; 50(1):104-15. [PubMed: 23523372]

29. Jiao X, et al. Identification of a quality-control mechanism for mRNA $5^{\prime}$-end capping. Nature. 2010; 467(7315):608-11. [PubMed: 20802481]

30. Dieci G, et al. Eukaryotic snoRNAs: a paradigm for gene expression flexibility. Genomics. 2009; 94(2):83-8. [PubMed: 19446021]

31. Filipowicz W, Pogacic V. Biogenesis of small nucleolar ribonucleoproteins. Curr Opin Cell Biol. 2002; 14(3):319-27. [PubMed: 12067654] 
32. Kawaji H, et al. Hidden layers of human small RNAs. BMC Genomics. 2008; 9:157. [PubMed: 18402656]

33. Ghosh A, Lima CD. Enzymology of RNA cap synthesis. Wiley Interdiscip Rev RNA. 2010; 1(1): 152-72. [PubMed: 21956912]

34. Shuman S. Capping enzyme in eukaryotic mRNA synthesis. Prog Nucleic Acid Res Mol Biol. 1995; 50:101-29. [PubMed: 7754031]

35. Yue Z, et al. Mammalian capping enzyme complements mutant Saccharomyces cerevisiae lacking mRNA guanylyltransferase and selectively binds the elongating form of RNA polymerase II. Proc Natl Acad Sci U S A. 1997; 94(24):12898-903. [PubMed: 9371772]

36. Schoenberg DR, Maquat LE. Re-capping the message. Trends Biochem Sci. 2009; 34(9):435-42. [PubMed: 19729311]

37. Burroughs AM, Aravind L. RNA damage in biological conflicts and the diversity of responding RNA repair systems. Nucleic Acids Res. 2016; 44(18):8525-8555. [PubMed: 27536007]

38. Lykke-Andersen J. Identification of a human decapping complex associated with hUpf proteins in nonsense-mediated decay. Mol Cell Biol. 2002; 22(23):8114-21. [PubMed: 12417715]

39. van Dijk E, et al. Human Dcp2: a catalytically active mRNA decapping enzyme located in specific cytoplasmic structures. EMBO J. 2002; 21(24):6915-24. [PubMed: 12486012]

40. Wang Z, et al. The hDcp2 protein is a mammalian mRNA decapping enzyme. Proc Natl Acad Sci U S A. 2002; 99(20):12663-8. [PubMed: 12218187]

41. Grudzien-Nogalska E, et al. Nudt3 is an mRNA decapping enzyme that modulates cell migration. RNA. 2016

42. Song MG, et al. Multiple mRNA decapping enzymes in mammalian cells. Mol Cell. 2010; 40(3): 423-32. [PubMed: 21070968]

43. Jurado AR, et al. Structure and function of pre-mRNA 5 ' -end capping quality control and $3{ }^{\prime}$-end processing. Biochemistry. 2014; 53(12):1882-98. [PubMed: 24617759]

44. Scheer H, et al. Uridylation Earmarks mRNAs for Degradation... and More. Trends Genet. 2016; 32(10):607-19. [PubMed: 27592415]

45. Malecki M, et al. The exoribonuclease Dis3L2 defines a novel eukaryotic RNA degradation pathway. EMBO J. 2013; 32(13):1842-54. [PubMed: 23503588]

46. Ustianenko D, et al. TUT-DIS3L2 is a mammalian surveillance pathway for aberrant structured non-coding RNAs. EMBO J. 2016; 35(20):2179-2191. [PubMed: 27647875]

47. Mullen TE, Marzluff WF. Degradation of histone mRNA requires oligouridylation followed by decapping and simultaneous degradation of the mRNA both $5^{\prime}$ to $3^{\prime}$ and $3^{\prime}$ to $5^{\prime}$. Genes Dev. 2008; 22(1):50-65. [PubMed: 18172165]

48. Song M, Kiledjian M. $3^{\prime}$ Terminal oligo U-tract-mediated stimulation of decapping. RNA. 2007; 13(12):2356-2365. [PubMed: 17942740]

49. Kiss T, Filipowicz W. Exonucleolytic processing of small nucleolar RNAs from pre-mRNA introns. Genes Dev. 1995; 9(11):1411-24. [PubMed: 7797080]

50. Yin QF, et al. Long noncoding RNAs with snoRNA ends. Mol Cell. 2012; 48(2):219-30. [PubMed: 22959273]

51. Topisirovic I, et al. Cap and cap-binding proteins in the control of gene expression. Wiley Interdiscip Rev RNA. 2011; 2(2):277-98. [PubMed: 21957010]

52. Lacerda R, et al. More than just scanning: the importance of cap-independent mRNA translation initiation for cellular stress response and cancer. Cell Mol Life Sci. 2017; 74(9):1659-1680. [PubMed: 27913822]

53. Turk EM, et al. The mitochondrial RNA landscape of Saccharomyces cerevisiae. PLoS One. 2013; 8(10):e78105. [PubMed: 24143261]

54. Xue Y, et al. Saccharomyces cerevisiae RAI1 (YGL246c) is homologous to human DOM3Z and encodes a protein that binds the nuclear exoribonuclease Rat1p. Mol Cell Biol. 2000; 20(11): 4006-15. [PubMed: 10805743]

55. Xiang S, et al. Structure and function of the $5^{\prime}-->3^{\prime}$ exoribonuclease Rat 1 and its activating partner Rai1. Nature. 2009; 458(7239):784-8. [PubMed: 19194460] 
56. Walters RW, et al. Edc3 function in yeast and mammals is modulated by interaction with NADrelated compounds. G3 (Bethesda). 2014; 4(4):613-22. [PubMed: 24504254]

57. Katsyuba E, Auwerx J. Modulating NAD(+) metabolism, from bench to bedside. EMBO J. 2017; 36(18):2670-2683. [PubMed: 28784597]

58. Haigis MC, Sinclair DA. Mammalian sirtuins: biological insights and disease relevance. Annu Rev Pathol. 2010; 5:253-95. [PubMed: 20078221]

59. Hentze MW, Preiss T. The REM phase of gene regulation. Trends Biochem Sci. 2010; 35(8):4236. [PubMed: 20554447]

60. Hentze MW, Kuhn LC. Molecular control of vertebrate iron metabolism: mRNA-based regulatory circuits operated by iron, nitric oxide, and oxidative stress. Proc Natl Acad Sci U S A. 1996; 93(16):8175-82. [PubMed: 8710843]

61. Wallander ML, et al. Molecular control of vertebrate iron homeostasis by iron regulatory proteins. Biochim Biophys Acta. 2006; 1763(7):668-89. [PubMed: 16872694]

62. Nagy E, Rigby WF. Glyceraldehyde-3-phosphate dehydrogenase selectively binds AU-rich RNA in the NAD(+)-binding region (Rossmann fold). J Biol Chem. 1995; 270(6):2755-63. [PubMed: 7531693]

63. Fulco M, et al. Glucose restriction inhibits skeletal myoblast differentiation by activating SIRT1 through AMPK-mediated regulation of Nampt. Dev Cell. 2008; 14(5):661-73. [PubMed: 18477450]

64. Canto $\mathrm{C}$, et al. AMPK regulates energy expenditure by modulating NAD+ metabolism and SIRT1 activity. Nature. 2009; 458(7241):1056-60. [PubMed: 19262508]

65. Canto C, et al. Interdependence of AMPK and SIRT1 for metabolic adaptation to fasting and exercise in skeletal muscle. Cell Metab. 2010; 11(3):213-9. [PubMed: 20197054]

66. Chen D, et al. Tissue-specific regulation of SIRT1 by calorie restriction. Genes Dev. 2008; 22(13): 1753-7. [PubMed: 18550784]

67. Kilberg MS, et al. Nutritional control of gene expression: how mammalian cells respond to amino acid limitation. Annu Rev Nutr. 2005; 25:59-85. [PubMed: 16011459]

68. Kiledjian, M., et al. Normal and Aberrantly Capped mRNA Decapping. In: Chanfreau, GFaTF., editor. The Enzymes: Eukaryotic RNases and their partners in RNA degradation and biogenesis. Academic Press; 2012. p. 165-180.

69. Talarek N, et al. Quantification of mRNA stability of stress-responsive yeast genes following conditional excision of open reading frames. RNA Biol. 2013; 10(8):1299-308. [PubMed: 23792549]

70. Mitchell SF, Parker R. Modifications on Translation Initiation. Cell. 2015; 163(4):796-8. [PubMed: 26544933]

71. Barvik I, et al. Non-canonical transcription initiation: the expanding universe of transcription initiating substrates. FEMS Microbiol Rev. 2017; 41(2):131-138. [PubMed: 27799279] 


\section{Trends}

Eukaryotic cells contain $\mathrm{NAD}^{+}$capped RNAs

$\mathrm{NAD}^{+}$cap can be added by both transcriptional initiation with $\mathrm{NAD}^{+}$in place of ATP, as well as a novel $\mathrm{NAD}^{+}$capping mechanism.

$5^{\prime}$ end $\mathrm{NAD}^{+}$cap promotes rapid decay of the RNA at least in part by the DXO family of proteins. 


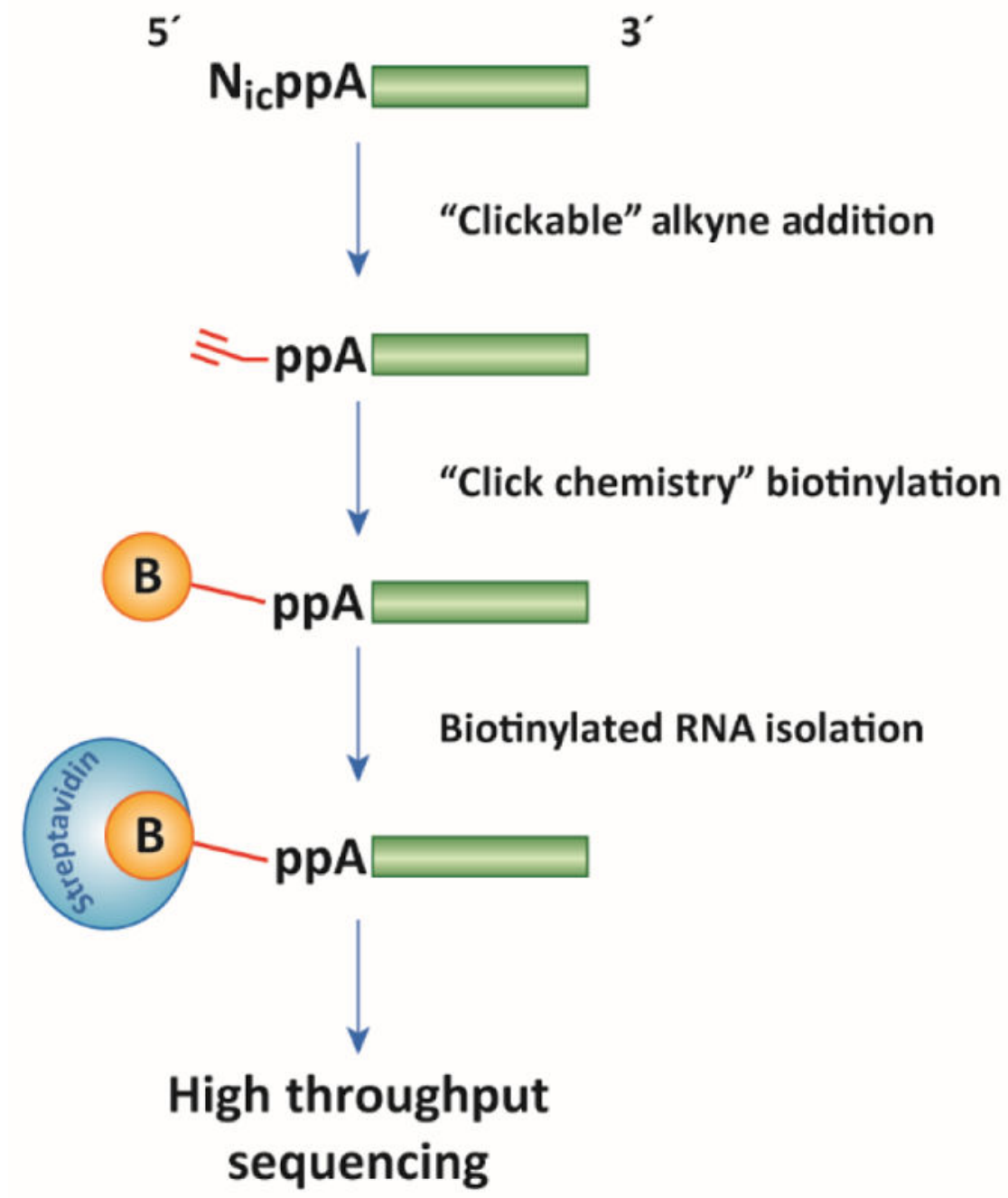

Figure 1. Schematic of NAD-captureSeq approach

An exchange of the nicotinamide moiety with an alkyne group is carried out with adenosine diphosphate-ribosylcyclase (ADPRC) and pentynol to attain the "clickable" alkyne group which is biotinylated by a copper catalyzed azide-alkyne cycloaddition reaction (click chemistry) and retained onto streptavidin beads. Identification of the captured RNAs are derived by high throughput RNA sequencing. See [20] for a detailed protocol. Nicotinamide is denoted as $\mathrm{N}_{\mathrm{ic}}$, the "p" represents organic monophosphate, the green bar indicates the RNA, the red lines are the alkyne group, the "B" denotes biotin and streptavidin is indicated. 
ATP

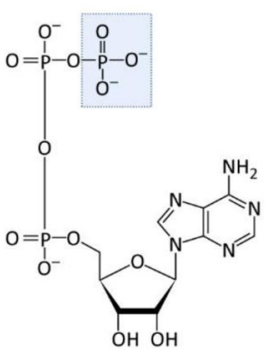

dpCoA

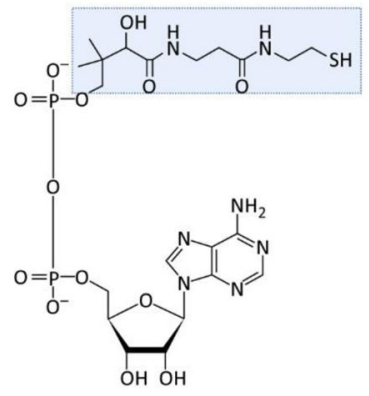

$\mathrm{NAD}^{+}$

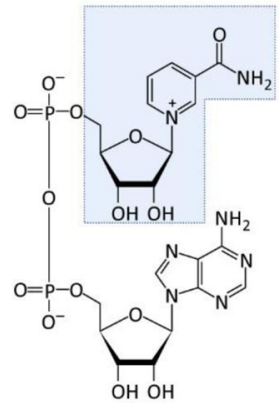

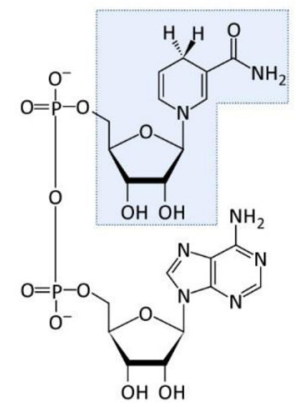

NADH

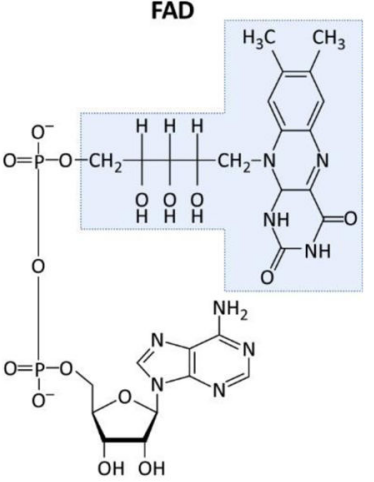

UDP-glucose

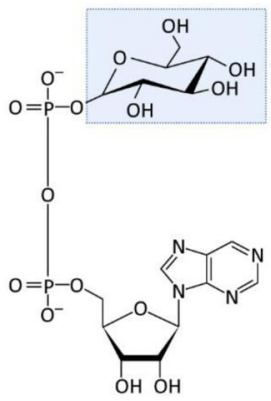

UDP-GICNAC

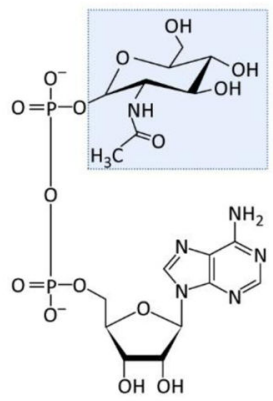

Figure 2. Adenosine-containing nucleoside metabolites

Structure of each metabolite is shown. All structures have a common adenosine diphosphate. The unique features of each metabolite are shaded in blue. 
(A)
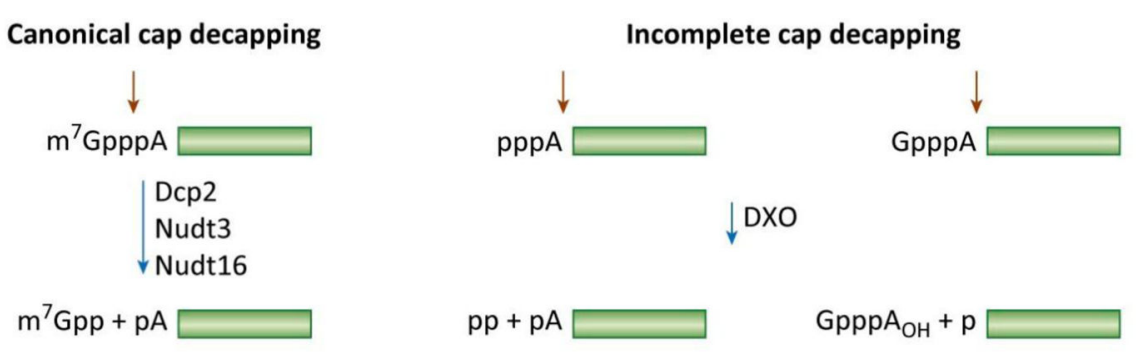

(B)

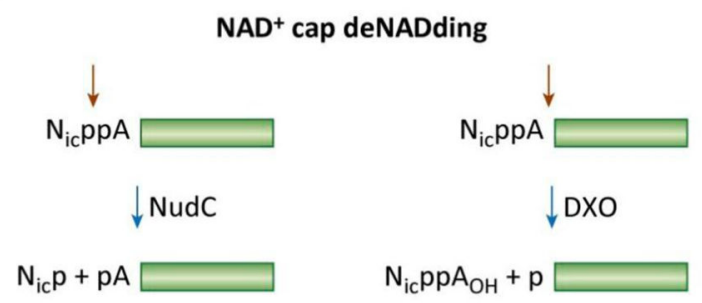

Figure 3. decapping and deNADding activities

(A) Canonical and incomplete cap decapping. Canonical $\mathrm{m}^{7} \mathrm{G}$-cap decapping activity by Dcp2, Nudt3 and Nudt16 decapping enzymes releasing $\mathrm{m}^{7} \mathrm{Gpp}$ and $5^{\prime}$ monophosphate RNA (Left). Non-canonical incomplete cap decapping activity by DXO removes either the diphosphate from a triphosphorylated RNA or the entire cap structure of an unmethylated capped RNA (Right). (B) deNADding activities of NudC and DXO are shown. NudC cleaves the $\mathrm{NAD}^{+}$cap between the two phosphates to release nicotinamide mononucleotide $\left(\mathrm{N}_{\mathrm{ic}} \mathrm{p}\right)$ and $5^{\prime}$ monophosphate RNA (Left). DXO hydrolyzes the phosphodiester linkage $3^{\prime}$ to the adenosine to remove the entire $\mathrm{NAD}^{+}$moiety and generate a $5^{\prime}$ monophosphate RNA (Right). Labeling is as in the legend to Figure 1 and the red arrow indicates the enzyme cleavage site. 

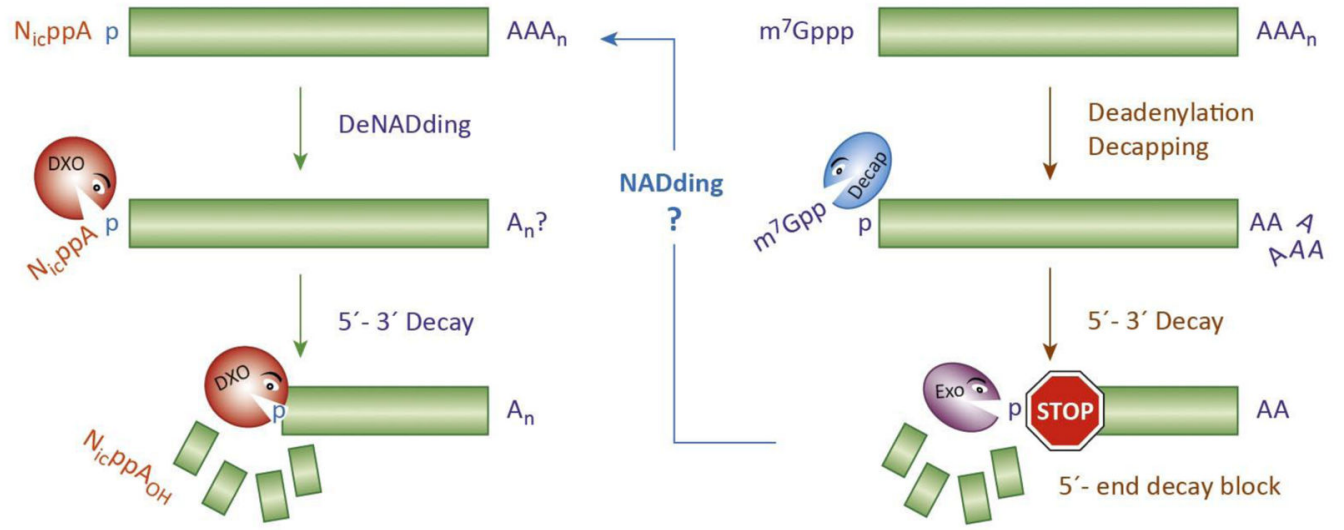

Figure 4. Model of DXO-mediated $\mathrm{NAD}^{+}$-capped mRNA decay

(Left) DXO can detect and remove the $\mathrm{NAD}^{+}$cap and degrade the mRNA by its intrinsic $5^{\prime}$ to $3^{\prime}$ exonucleolytic activity. The polyadenylated tail is shown as $\mathrm{AAA}_{n}$ with the " $\mathrm{A}_{\mathrm{n}}$ ?" representing the unknown parameter of whether the tail is deadenylated to an oligoadenylated tail prior to deNADding. The DXO enzyme is as shown. Labeling is as in the legend to Figure 1. (Right) Decay of $\mathrm{m}^{7} \mathrm{G}$ capped RNA by decapping (blue) and $5^{\prime}$ exonuclease (brown) is shown. The Stop sign denotes a stable $5^{\prime}$ end decay intermediate refractory to exonucleolytic decay. The potential NADding of the stable intermediate to further promote decay is represented by the blue arrow and question mark. 